\section{SYKing inhibitors on retinoblastoma}

\section{By Chris Cain, Staff Writer}

Findings from St. Jude Children's Research Hospital and Washington University in St. Louis researchers show that spleen tyrosine kinase is epigenetically upregulated in retinoblastoma, suggesting that blocking its activity could help treat the disease. ${ }^{1}$

The results provide a potential new indication for spleen tyrosine kinase inhibitors, and the researchers are already working on an ocular formulation of one.

Retinoblastoma is a rare childhood cancer of the eye most commonly caused by inactivating mutations in the retinoblastoma 1 (RB1) tumor suppressor gene. Although more than $95 \%$ of children in the U.S. are cured of the disease, treatment requires chemotherapy or localized radiation therapy, and patients who do not adequately respond are at risk for vision loss. In developing nations the prognosis is worse, as the cancer can metastasize if not caught early.

In 2010, St. Jude and Washington University formed the Pediatric Cancer Genome Project, a three-year collaboration to sequence the genomes of about 600 pediatric cancer patients, including those with retinoblastoma.

Because RB1 is a known regulator of essential cellular processes including DNA methylation, histone modification and cell cycle progression, researchers involved in the project suspected they would find genetic changes that might point the way to new targets.

Surprisingly, the team did not find a striking amount of genetic change in the four sequenced primary retinoblastoma samples or in an orthotopic retinoblastoma xenograft that had grown in mice for more than nine months. The rates of mutation and chromosomal structural alterations were lower than those seen in many other tumor types.

The lack of genetic changes prompted the researchers to look more closely at genomewide epigenetic changes, including histone and DNA methylation patterns, to help explain how loss of $R B 1$ drives retinoblastoma growth.

The team combined genomewide expression data with an analysis of histone modification and DNA methylation to pinpoint candidate genes that had significant changes in patient samples compared with normal fetal retinas. Spleen tyrosine kinase (SYK) came out of the integrated computational analysis as the top kinase hit.
"SYK was upregulated in the gene expression analysis, but it was not our top candidate until we did the complete epigenetic analysis," said Michael Dyer, a senior author of the study.

He said SYK is expressed in blood cells that often contaminate tumor samples and is not normally expressed in the eye. Thus, the team would have considered it a false positive without the epigenetic data to back it up.

Dyer is coleader of the Developmental Therapeutics for Solid Malignancies Program and director of the Division of Developmental Biology at St. Jude.

The group next assessed the functional role of SYK in retinoblastoma. SYK is normally expressed in immune cells and is a regulator of inflammation, but it has also been suggested as a target in hematological malignancies because it can promote cell survival.

In cultured retinoblastoma cells, small hairpin RNA knockdown or pharmacological inhibition of SYK induced cell death compared with no knockdown or treatment. In an orthotopic xenograft model of retinoblastoma, topotecan plus ocular injection of a SYK inhibitor prolonged survival compared with topotecan alone.

Results were published in Nature.

"The beauty of this story is that because there were hardly any genetic lesions, we could dig into the epigenetic analysis and look for specific changes. It would have been very hard to sort out what the contributing genetic and epigenetic factors were if you had thousands of point mutations and hundreds of chromosomal translocations on top of these epigenetic changes," said Dyer.

Joan O'Brien, chair of the Department of Ophthalmology at the Scheie Eye Institute of the University of Pennsylvania, told SciBX, "The preclinical data provided here are indeed suggestive that SYK inhibition could play a clinical role in treatment of retinoblastoma. More specific targeted therapies are required for this disease because neonatal patients with germline retinoblastoma experience an increased incidence of second tumors as well as side effects from today's treatment regimens."

She added that the lack of genetic variation in these samples is a surprising finding and further studies are needed to reconcile this study with previous work. Previous studies in cell culture had also suggested loss of $R B 1$ could lead to widespread chromosomal structural alterations. $^{2}$

Dyer said experiments are ongoing to explain the apparent discrepancy between those studies and what his team found in primary tumor samples.

David Cobrinik, a senior research scientist at Memorial SloanKettering Cancer Center, said that sequencing additional samples is necessary to confirm that there is consistently a low level of genomic structural alteration. He also cautioned that the high levels of SYK could reflect SYK expression in a rare retinal cell type, as well as the adoption of an altered epigenetic state.

"We are very up front in the paper that we have no idea what the mechanism of SYK upregulation is," Dyer told SciBX. He said RB1 could 


\section{ANALYSIS}

\section{TARGETS \& MECHANISMS}

be acting to directly regulate $S Y K$ transcription or acting indirectly by globally changing chromatin structure.

Dyer's lab is now studying the pathways upstream and downstream of SYK in retinoblastoma and is investigating SYK expression in metastatic retinoblastoma samples.

\section{Targeting SYK}

Dyer also is developing an ocular formulation of Rigel Pharmaceuticals Inc.'s R406 small molecule SYK inhibitor, which is available as a research reagent. His group has developed a pharmacokinetics assay to detect R406 in the plasma, retina and vitreous of the eye and plans to also examine ocular toxicity.

Dyer has no specific timeline for starting a Phase I trial of the formulation.

Rigel's fostamatinib (R788), an oral prodrug formulation of R406 that is partnered with AstraZeneca plc, is in Phase III testing to treat rheumatoid arthritis (RA) and in Phase II trials to treat diffuse large B cell lymphoma (DLBCL).

"AstraZeneca is committed to developing a greater understanding of SYK in a range of pathologies," but the pharma has no plans for clinical studies in other disease areas at this time, according to spokesperson Sameena Conning.

Other SYK inhibitors in clinical development include the oral small molecules PRT062607 from Portola Pharmaceuticals Inc. and partner Biogen Idec Inc., and Gilead Sciences Inc.'s GS-9973. PRT062607 has completed Phase I testing, and the partners plan to begin a Phase IIa trial in RA in 2H12. GS-9973 is in Phase I testing for RA. The three companies declined to comment on the Nature results.

Also in development is ZaBeCor Pharmaceutical Co.'s Excellair small interfering RNA targeting SYK, which has completed a Phase II trial to treat asthma.

ZaBeCor founder, chairman and CEO Alan Schreiber told SciBX that studies have shown inhibiting SYK is effective in preventing cancer cell proliferation and noted that siRNA could be an effective way to deliver therapeutics to the eye. He said the company has performed preclinical studies demonstrating the use of siRNA to inhibit SYK activity in mouse models of ocular inflammation.
ZaBeCor owns U.S. and international patents covering the use of its SYK-targeting siRNA technology to target any human organ. The company is looking for partners to develop its technology in retinoblastoma and other indications.

In addition to his work on SYK, Dyer is developing two other treatments for retinoblastoma targeting distinct pathways altered by loss of $R B 1$.

Changes in genomewide histone acetylation linked to loss of $R B 1$ have suggested that patients with retinoblastoma may benefit from treatment with histone deacetylase (HDAC) inhibitors. ${ }^{3}$ Separately, overexpression of the survival factors $\mathrm{mdm} 2 \mathrm{p} 53$ binding protein homolog (MDM2; HDM2) and MDM4 (MDMX) in patients with retinoblastoma suggested a use for MDM inhibitors. ${ }^{4}$

"We have three possible approaches here, so we're going to get all the data and sit down and decide how to proceed based on efficacy, toxicity and route to the clinic," Dyer said. He added that once the data package is assembled, he wants to look for corporate partnerships.

The findings reported in Nature are unpatented.

Cain, C. SciBX 5(7); doi:10.1038/scibx.2012.168

Published online Feb. 16, 2012

\section{REFERENCES}

1. Zhang, J. et al. Nature; published online Jan. 11, 2012; doi:10.1038/nature10733

Contact: Michael A. Dyer, St. Jude Children's Research Hospital, Memphis, Tenn.

e-mail: michael.dyer@stjude.org

2. Manning, A.L. et al. Genes Dev. 24, 1364-1376 (2010)

3. Dalgard, C.L. et al. Clin. Cancer Res. 14, 3113-3123 (2008)

4. Brennan, R.C. et al. Cancer Res. 71, 4205-4213 (2011)

COMPANIES AND INSTITUTIONS MENTIONED

AstraZeneca plc (LSE:AZN; NYSE:AZN), London, U.K. Biogen Idec Inc. (NASDAQ:BIIB), Weston, Mass. Gilead Sciences Inc. (NASDAQ:GILD), Foster City, Calif. Memorial Sloan-Kettering Cancer Center, New York, N.Y. Portola Pharmaceuticals Inc., South San Francisco, Calif. Rigel Pharmaceuticals Inc. (NASDAQ:RIGL), South San Francisco, Calif.

St. Jude Children's Research Hospital, Memphis, Tenn. University of Pennsylvania, Philadelphia, Pa. Washington University in St. Louis, St. Louis, Mo. ZaBeCor Pharmaceutical Co., Bala Cynwyd, Pa. 